\title{
Port-synchronizable test sequences for communication protocols
}

\author{
K. C. Tai ${ }^{*}$ and Y.C. Young ${ }^{*} *$ \\ Department of Computer Science, North Carolina State University \\ Raleigh, North Carolina, 27695-8206, USA \\ Tel: (919) 515-7146, Fax: (919) 515-7896 \\ e-mail: kct@csc.ncsu.edu, yyoung@vnet.ibm.com
}

\begin{abstract}
In conformance testing of a communication protocol, the synchronization between inputs from different testers for the protocol becomes a problem. A synchronizable test sequence of a finite state machine (FSM) is a test sequence for which the synchronization problem either does not exist or can be solved by communication between testers. In this paper, for a multi-port FSM with one tester for each port, we define a necessary and sufficient condition under which a test sequence of the FSM does not have the synchronization problem. Such a test sequence is called a port-synchronizable test sequence. Our empirical results show that an FSM may contain many port-synchronizable test sequences that are not synchronizable according to a previous definition of a synchronizable test sequence.
\end{abstract}

\section{Keywords}

Protocol testing, synchronizable test sequences, finite state machines

* This work was supported in part by the US National Science Foundation under grant CCR9309043.

\# The author is also with IBM, Raleigh, North Carolina. 


\section{INTRODUCTION}

The finite state machine (FSM) model is commonly used for specifying communication protocols. The problem of generating test sequences based on an FSM has been studied for about two decades (Tarnay, 1991) (Sarikaya, 1993). When an implementation of an FSM is tested for conformance, test sequences are derived from the FSM, and testers (or test drivers) for the implementation are constructed according to these test sequences. With the use of multiple testers for an FSM, the synchronization between inputs from different testers becomes a problem. A synchronizable test sequence of an FSM is a test sequence for which the synchronization problem either does not exist or can be solved by communication between testers. In (Sarikaya and Bochmann, 1984) the synchronization problem for an FSM with two testers was discussed, and a type of synchronizable test sequence that does not have the synchronization problem was defined. In recent years, several issues on synchronizable test sequences have been studied.

Due to the existence of distributed database systems and communication networks, FSMs with multiple ports are needed to specify protocols (Luo et al., 1993). Also, the use of multi-port FSMs makes the design of communication protocols flexible. Protocol specification languages such as LOTOS, Estelle, and SDL (Turner, 1993) allow the use of multiple ports. In this paper we define a necessary and sufficient condition under which a test sequence of an FSM with multiple ports does not have the synchronization problem. Such a test sequence is called a portsynchronizable test sequence. Based on our new definition, more test sequences of an FSM become synchronizable.

This paper is organized as follows. Section 2 provides basic definitions. Section 3 summarizes previous work on synchronizable test sequences of an FSM. Section 4 gives our motivation for extending the definition of a synchronizable test sequence in (Sarikaya and Bochmann, 1984). Section 5 shows how to construct a set of testers from a test sequence, based on the assumption of port-based testing, which does not allow different testers for an FSM to communicate with each other. Also, section 5 defines the port-based synchronization problem. Section 6 gives the definition of a port-synchronizable test sequence of an FSM and shows that this definition provides a necessary and sufficient condition under which a test sequence of an FSM does not have the port-based synchronization problem. Section 7 discusses the generation of port-synchronizable test sequences of an FSM. Section 8 shows the results of our empirical studies on synchronizable test sequences. Section 9 concludes this paper.

\section{PRELIMINARIES}

Below we provide a formal definition of a multi-port FSM, which is different from that in (Luo et al., 1993).

Definition. A finite state machine (FSM) $M$ with multiple ports is defined as a 6-tuple $\mathrm{M}=(\mathrm{S}, \mathrm{I}, \mathrm{O}, \mathrm{T}, \mathrm{U}, \mathrm{s} 0)$, where

- $\mathrm{S}$ is the set of states of $\mathrm{M}$ 
- I is the set of input symbols of M. Each input symbol is of the form P:A, where P denotes a port and $\mathrm{A}$ an input message.

- $\mathrm{O}$ is the set of output symbols of $M$. Each output symbol is of the form $\mathrm{Q}: \mathrm{B}$, where $\mathrm{Q}$ denotes a port and $B$ an output message.

- $T$ is the transition function of $M$, which maps from $D$ to $S$, where $D \subseteq S \times I$. (Thus, $M$ is deterministic.)

- $\mathrm{U}$ is the output function of $\mathrm{M}$, which maps from $\mathrm{D}$ to $(\mathrm{O} 1 \times \mathrm{O} 2 \times \ldots \times \mathrm{Ov}) \cup\{\varepsilon\}$, where each Oi, $1<=\mathrm{i}<=\mathrm{v}$, is an output symbol, and $\varepsilon$ stands for the empty output. (The output of a transition may contain two or more output symbols with the same port name.)

- $\mathrm{s} 0$ is the initial state of M.

In the above definition, each input or output symbol contains two elements: port name and message. The separation of port name and message makes it easier to discuss synchronizable test sequences. If the transition function of an FSM allows a state to have a transition on an input, then this input is said to be valid for the state. The output function of an FSM allows zero, one, or more output symbols to be associated with a transition. An FSM communicates with its environment by receiving input symbols and sending output symbols via ports. Each port of an FSM has two unbounded FIFO queues: the input queue, which keeps input messages of the FSM, and the output queue, which keeps output messages of the FSM. It is assumed that the delivery of messages from an FSM to any of its port is FIFO, i.e., messages sent from $M$ to the same port are received in the order sent. For an FSM with two ports, its two testers are commonly referred to as the lower and upper testers. In this paper, the lower and upper testers are referred to as the $\mathbf{L}$-tester and the $\mathbf{U}$-tester, respectively and the two ports connected to the $\mathrm{L}$ - and U-testers are referred to as ports $\mathbf{P}_{\mathbf{L}}$ and $\mathbf{P}_{\mathrm{U}}$, respectively.

Assume that during an execution of an FSM and its environment, the current state of the FSM is S. For a transition T of S, if the first input message at the input port of $\mathrm{S}$ is valid for $\mathrm{S}$, then $\mathrm{T}$ is said to be eligible for $\mathrm{S}$. If $\mathrm{S}$ has two or more eligible transitions, then one of them is chosen at random for execution. If $S$ does not have any eligible transitions, then the FSM waits until at least one eligible transition for $\mathrm{S}$ becomes available.

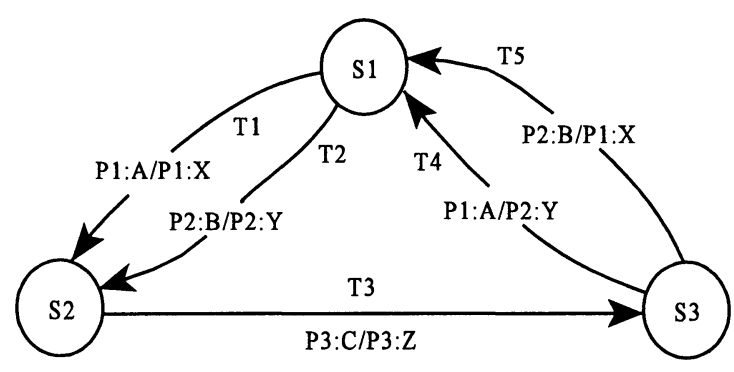

Figure. 1. FSM M1 with Ports P1, P2, and P3 
A transition $\mathrm{T}$ from state $\mathrm{Si}$ to state $\mathrm{Sj}$ that has $\mathrm{P}: \mathrm{A}$ as the input symbol and $(\mathrm{Q} 1: \mathrm{B} 1, \mathrm{Q} 2: \mathrm{B} 2, \ldots, \mathrm{Qv}: \mathrm{Bv})$ as the sequence of output symbols is denoted as $(\mathrm{Si}, \mathrm{Sj}$, $\mathrm{P}: \mathrm{A} /(\mathrm{Q} 1: \mathrm{B} 1, \mathrm{Q} 2: \mathrm{B} 2, \ldots, \mathrm{Qv}: \mathrm{Bv})$, and $\mathrm{Si}$ is referred to as the head state of $\mathrm{T}$ or head(T), $\mathrm{Sj}$ the tail state of $\mathrm{T}$ or tail(T), $\mathrm{P}$ the input port of $\mathrm{T}$, $\mathrm{A}$ the input message of $\mathrm{T}, \mathrm{Qi}, 1<=\mathrm{i}<=\mathrm{v}$, an output port of $\mathrm{T}$, and $\mathrm{Bi}$ an output message of $\mathrm{T} . \mathrm{P}: \mathrm{A} /(\mathrm{Q} 1: \mathrm{B} 1, \mathrm{Q} 2: \mathrm{B} 2, \ldots, \mathrm{Qv}: \mathrm{Bv})$ is referred to as the label of $T$ or label $(T)$. For the sake of simplicity, if $v=1, P: A /(Q 1: B 1)$ is referred to as $\mathrm{P}: \mathrm{A} / \mathrm{Q} 1: \mathrm{B} 1$. If $\mathrm{T}$ has no output symbols, it is denoted as $(\mathrm{Si}, \mathrm{Sj}, \mathrm{P}: \mathrm{A} / \boldsymbol{\varepsilon})$. A port is said to be involved in a transition $\mathrm{T}$ if this port is either the input port or an output port of $\mathrm{T}$.

For an FSM M, let $D_{M}=\left(V_{M}, E_{M}\right)$ denote the digraph of $M$, where $V_{M}$ is a set of vertices, each representing a state of $M$, and $E_{M}$ is a set of directed edges, each representing a transition of M. Figure 1 shows the digraph of FSM M1, which has three ports P1, P2, and P3, and three states $\mathrm{S} 1, \mathrm{~S} 2$ and $\mathrm{S} 3$, with $\mathrm{S} 1$ being the initial state. A, B and $\mathrm{C}$ are input messages of ports $\mathrm{P} 1$, $\mathrm{P} 2$ and $\mathrm{P} 3$, respectively, and $\mathrm{X}, \mathrm{Y}$ and $\mathrm{Z}$ are output messages of ports $\mathrm{P} 1, \mathrm{P} 2$ and $\mathrm{P} 3$, respectively. A tabular representation of $\mathrm{M} 1$ is given below, which has inputs as rows and states as columns.

\begin{tabular}{llcc} 
& \multicolumn{1}{c}{ S1 } & S2 & S3 \\
P1:A & P1:X, S2 (T1) & & P2:Y, S1 (T4) \\
P2:B & P2:Y, S2 (T2) & & P1:X, S1 (T5) \\
P3:C & & P3:Z, S3 (T3) &
\end{tabular}

Each non-empty entry in the above table defines a transition for the corresponding state and input symbol; it contains the output symbol and tail state of this transition, followed by a pair of parentheses enclosing the name of this transition.

Let $\mathrm{M}$ denote an FSM. A transition sequence of $M$ is a sequence of consecutive transitions in $M$, and a test sequence of $M$ is a transition sequence of $M$ starting from the initial state of $M$. In this paper, a transition or test sequence is often denoted as a sequence of transition names connected by ".". For example, T1.T3.T5 and T4.T2.T3 are transition sequences of M1 in Figure 1 , and the former is a test sequence of $\mathrm{M} 1$, but the latter is not. For a transition sequence, its $\mathrm{i} / \mathrm{o}$ sequence refers to the sequence of labels associated with the transitions in the transition sequence. Since a test sequence of $M$ starts with the initial state of $M$, it does not need to keep information about the head and tail states of transitions in the test sequence. Thus, a test sequence of $\mathrm{M}$ can be denoted by its $\mathrm{i} / \mathrm{o}$-sequence. For example, T1.T2.T3 of $\mathrm{M}$ can be denoted by its i/o sequence is (P1:A/P1:X, P3:C/P3:Z, P2:B/P1:X). Similarly, a transition sequence of $\mathrm{M}$ that starts from a given state can be denoted by its $\mathrm{i} / \mathrm{o}$ sequence. For a transition sequence $\mathrm{E}$ of an FSM, if a transition T occurs (immediately) before a transition T' in $E$, then $T$ is said to be a (the immediate) predecessor of T' in $E$ and T' a (the immediate) successor of $T$ in $E$.

A digraph $D=(V, E)$ is strongly connected if for every pair of vertices $\mathrm{Vi}$ and $\mathrm{Vj}$ in $\mathrm{V}$, there exists a path from $\mathrm{Vi}$ to $\mathrm{Vj}$. An FSM is said to be strongly connected if its digraph is strongly connected. An FSM is said to be completely specified if each state of this FSM has a transition for every possible input symbol. For a state $\mathrm{S}$ of an FSM, let IO(S) be the set of i/o sequences that start from S. An FSM is said to be minimal if for any two states S and S' of the FSM, IO(S) $\neq \mathrm{IO}\left(\mathrm{S}^{\prime}\right)$. In this paper, we assume that each FSM is deterministic, strongly connected, minimal, 
and possibly incompletely specified, unless otherwise specified.

A postman tour (or transition tour) of a digraph $D=(V, E)$ is a path in $D$ that starts and ends at the same vertex and covers each edge in $E$ at least once. A postman tour of an FSM is a postman tour in the digraph of this FSM. A digraph D has a postman tour if and only if D is strongly connected.

For a state $\mathrm{S}$ in an FSM M, a unique input/output (UIO) sequence $\mathrm{E}$ is a transition sequence starting from $S$ such that if the sequence of input symbols of $E$ is applied to a state in $M$ other than $\mathrm{S}$, the sequence of corresponding output symbols is different from the sequence of output symbols of $\mathrm{E}$. In other words, an UIO sequence for $\mathrm{S}$ can distinguish $\mathrm{S}$ from other states in $\mathrm{M}$. The UIO- or U-method for testing an FSM verifies the tail state of a transition of the FSM by using an UIO sequence for the tail state (Sabnani and Dahbura, 1988).

\section{PREVIOUS WORK ON SYNCHRONIZABLE TEST SEQUENCES OF AN FSM}

(Sarikaya and Bochmann, 1984) considered the synchronization problem for an FSM with two ports (for the L- and U-testers, which do not communicate with each other). Two consecutive transitions are said to have the synchronization problem if the input port of the second transition is not involved in the first transition (i.e., the input port of the second transition is neither the input port nor an output port of the first transition.) A test sequence is said to be synchronizable if no two consecutive transitions of the test sequence have the synchronization problem. (Sarikaya and Bochmann, 1984) also discussed how to extend existing test sequence generation methods in order to generate synchronizable test sequences. In order to distinguish the type of synchronizable test sequence defined in (Sarikaya and Bochmann, 1984) from other types of synchronizable test sequences, the former is referred to as a pair-synchronizable test sequence in the remainder of this paper.

(Chen et al., 1990) defined a tightly synchronizable test sequence of a two-port FSM as a test sequence such that for any two consecutive transitions, the input port of the second transition is the same as the output port of the first transition. (Each transition was assumed to have at most one output symbol.) They showed how to construct a graph, called the duplex digraph, of a two-port FSM such that a test sequence of the duplex graph is a tightly synchronizable test sequence of the FSM.

(Boyd and Ural, 1991) investigated complexity issues related to pair-synchronizable test sequences of a two-port FSM. They presented a necessary and sufficient condition for the existence of a pair-synchronizable postman tour of an FSM, and this condition can be determined in polynomial time. Also, they showed that the problem of finding a minimum-length pairsynchronizable postman tour of an FSM is NP-complete.

(Ural and Wang, 1993) considered two-port FSMs satisfying a number of conditions, including the necessary and sufficient condition in (Boyd and Ural, 1991) and the condition that each state in the FSM possess two UIO sequences, one for the L-tester and the other for the Utester. By modifying the algorithm in (Chen et al., 1990) for the construction of a duplex digraph 
and by providing additional algorithms, they showed how to find a pair-synchronizable UIObased test sequence of an FSM in polynomial time. (Guyot and Ural , 1995) extended the work by showing the construction of a digraph of an FSM M such that all paths in the digraph are pairsynchronizable test sequences of $\mathrm{M}$ and by showing that under certain conditions, a pairsynchronizable test sequence of $\mathrm{M}$ can distinguish $\mathrm{M}$ from any FSM not isomorphic to $\mathrm{M}$.

(Chen and Ural, 1995) allowed the L- and U-testers for a two-port FSM to communicate with each other, and they considered the cost of such communication in test sequence generation. They showed how to convert a non-pair-synchronizable test sequence into a synchronizable test sequence by adding communication statements between the L- and U-testers. Such synchronizable test sequences are referred to as $\mathbf{L U}$-synchronizable test sequences in this paper. Based on the duplexU digraph of an FSM, a minimum-cost LU-synchronizable test sequence using multiple UIO sequences can be generated. Since the generation of such a test sequence is an NP-complete problem, they proposed a heuristic algorithm that yields an LUsynchronizable UIO-based test sequence with its cost within a bound of the minimum cost.

(Luo et al., 1993) discussed the need for FSMs with multiple ports and extended the definition of a pair-synchronizable test sequence for a multi-port FSM. They also investigated the issue of fault coverage by pair-synchronizable test sequences.

The use of synchronizable test sequences is necessary for conformance testing of an implementation of an FSM, since the implementation is treated as a black box. When the implementation is tested by its developers, it can be modified to perform deterministic testing and thus make every test sequence synchronizable. Details on deterministic testing and debugging of concurrent programs can be found in (Tai and Ahuja, 1987) (Carver and Tai, 1991) (Tai et al., 1991) (Tai and Carver, 1995).

\section{MOTIVATION FOR EXTENDING THE DEFINITION OF A PAIR- SYNCHRONIZABLE TEST SEQUENCE}

In this section, we show that the definition of a pair-synchronizable test sequence is more restrictive than necessary and that some non-pair-synchronizable test sequences are actually synchronizable. Below we first give a general definition of a synchronizable test sequence.

Definition. A test sequence for an FSM M is said to be synchronizable if any execution of $\mathrm{M}$ and the testers generated according to the test sequence is deterministic (i.e., at any time during an execution of $\mathrm{M}$ and these testers, the current state of $\mathrm{M}$ has at most one eligible transition).

Notes:

- In this paper, the issue of fault detection is not discussed. Therefore, we consider executions of $\mathrm{M}$ and its testers, not executions of an implementation of $M$ and its testers.

- Whether a test sequence of $M$ is synchronizable depends on the test sequence, $M$, and the construction of testers for the test sequence.

According to (Sarikaya and Bochmann, 1984), two consecutive transitions are said to have the 
synchronization problem if the input port of the second transition is not involved in the first transition. In the remainder of this paper, this synchronization problem is referred to as the pairbased synchronization problem. To illustrate this problem for a two-port FSM, we first show how the L- and U-testers work for two consecutive transitions T1 and T2. Assume that the input port of $T 2$ is port $P_{L}$. The following are three possible relationships between port $P_{L}$ and $T 1$ :

(a) Port $P_{L}$ is an output port of T1. In this case, the L-tester sends the input message of T2 to port $\mathrm{P}_{\mathrm{L}}$ immediately after receiving the output message of $\mathrm{T} 1$ from port $\mathrm{P}$. No synchronization problem exists.

(b) Port $P_{L}$ is not an output port of $T 1$, but is the input port of T1. In this case, the L-tester sends the input message of $T 2$ to port $P_{L}$ immediately after sending the input message of $\mathrm{T} 1$ to port $\mathrm{P}_{\mathrm{L}}$. Thus, the input message of $\mathrm{T} 2$ will be received after the completion of $\mathrm{T} 1$. No synchronization problem exists.

(c) Port $P_{L}$ is neither the input port nor an output port of T1 (i.e., only port $P_{U}$ is involved in T1). In this case, the L-tester sends the input message of $T 2$ to port $P_{L}$ without any dependency on T1. According to (Sarikaya and Bochmann, 1984), this case creates the pair-based synchronization problem.

Now we examine the effect of case (c). Let S1 and S2 be the head and tail states of T1, respectively. Assume that $\mathrm{S} 1$ is the current state, the input message of T2 is the first input message at port $P_{L}$, and the input message of $T 1$ is the first input message at port $P_{U}$. Also, we assume that $\mathrm{S} 1$ contains a transition, say $\mathrm{T} 3$, that has the same input port and message as $\mathrm{T} 2$ (see below).

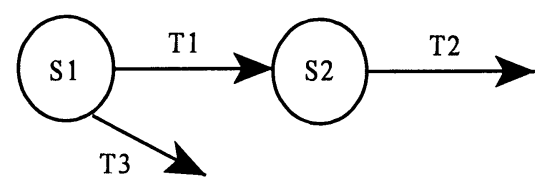

Since the first input messages at both ports $\mathrm{P}_{\mathrm{L}}$ and $\mathrm{P}_{\mathrm{U}}$ are valid for $\mathrm{S} 1$, both transitions $\mathrm{T} 1$ and T3 are eligible for $\mathrm{S} 1$. If the first input message at port $\mathrm{P}_{\mathrm{L}}$ is accepted (i.e., transition T3 is executed), then a synchronization problem occurs, since $T 1$, not $T 3$, is expected to be executed. To prevent this problem to occur, the definition of a pair-synchronizable test sequence in (Sarikaya and Bochmann, 1984) does not allow case (c). As a result, at any time during an execution of a two-port FSM by using a pair-synchronizable test sequence, at most one of ports $\mathrm{P}_{\mathrm{L}}$ and $\mathrm{P}_{\mathrm{U}}$ contains input messages.

Now we assume that transition $\mathrm{T} 3$ does not exist (i.e., state $\mathrm{S} 1$ does not contain any transition with the same input symbol as T2). When S1 is the current state, the arrival of the input message of $T 2$ at port $P_{L}$ does not create the pair-based synchronization problem since the input symbol of $\mathrm{T} 2$ is invalid for $\mathrm{S} 1$. Therefore, we can allow both ports $\mathrm{P}_{\mathrm{L}}$ and $\mathrm{P}_{\mathrm{U}}$ to contain input messages as long as only one eligible transition exists for the current state. Following the above discussion, the definition of a pair-synchronizable test sequence is more restrictive than necessary, and some 
non-pair-synchronizable test sequences are actually synchronizable. Note that the definition of a pair-synchronizable test sequence is based solely on the test sequence. Our new concept of a synchronizable test sequence of an FSM is based on not only the test sequence, but also the transitions of the states in the FSM that are passed through by the test sequence.

Since the multi-port FSM model becomes more commonly used and is more general than the two-port FSM model, we define our new synchronizable test sequences for the multi-port FSM model. In the remainder of this paper, an FSM is assumed to have multiple ports, unless otherwise specified. There are two testing strategies for an FSM: port-based testing, which does not allow testers for the FSM to communicate with each other, and group-based testing, which divides the ports of the FSM into groups and allows the testers for ports in the same group to communicate with each other. In this paper, we focus on port-based testing.

\section{PORT-BASED TESTERS AND SYNCHRONIZATION PROBLEM}

In this section, we first show the generation of a set of testers from a test sequence, according to port-based testing. Such testers are referred to as port-based testers. We then define the portbased synchronization problem, according to port-based testers..

The port-based tester for port $\mathrm{P}$ of an FSM M according to a test sequence $\mathrm{E}$ of $\mathrm{M}$, denoted as Tester(P,E,M), contains the following two types of statements:

- send A to P;

- receive $\mathrm{B}$ from $\mathrm{P}$;

where $\mathrm{A}$ is an input message of $\mathrm{M}, \mathrm{B}$ is an output message of $\mathrm{M}$, a send operation is nonblocking, and a receive operation is blocking. For the sake of simplicity, Tester(P,E,M) is referred to as Tester(P) if $E$ and $M$ are either implied or immaterial, and as Tester(P,E) if $M$ is implied. When "receive B from $P$ " is executed by Tester(P), if the first output message of $M$ at port $\mathrm{P}$ is not $\mathrm{B}$, then Tester $(\mathrm{P})$ has an abnormal termination. It is assumed that the delivery of messages from Tester(P) to port $\mathrm{P}$ is FIFO, i.e., messages sent from Tester(P) to port $\mathrm{P}$ are received in the order sent. In section 2, the FSM model assumes that each transition is associated with an input symbol and zero, one, or more output symbols. Thus, a transition in an FSM can be viewed as a receive statement, followed by zero, one, or more send statement. For the sake of simplicity, a tester is defined as a sequence of send and receive statements, not as a sequence of transitions. If necessary, a sequence of send and receive statements can be converted into a sequence of transitions.

\section{Algorithm Port_Tester_Gen}

Input: a test sequence $\mathrm{E}$ of an FSM M

Output: Tester(E), which is $\{$ Tester(P,E) $\mid \mathrm{P}$ is a port involved in $\mathrm{E}\}$

(1) For each port $P$ involved in $E$, let Tester $(P, E)$ be empty.

(2) For each transition $T$ in $E$ (starting from the first transition in $E$ ),

(a) if the input symbol of T is $\mathrm{P}: \mathrm{A}$, then add "send $\mathrm{A}$ to $\mathrm{P}$ " to the end of Tester(P,E).

(b) if the list of output symbols of $\mathrm{T}$ is $(\mathrm{Q} 1: \mathrm{B} 1, \mathrm{Q} 2: \mathrm{B} 2, \ldots, \mathrm{Qv}: \mathrm{Bv})$, then 
for each i from 1 to $\mathrm{v}$, add "receive Bi from Qi" to the end of Tester(Qi,E). end of algorithm.

Consider test sequence R1 = T1.T3.T4 of M1, which is shown in Figure 1. By applying the above algorithm to T1.T3.T4, Tester(R1) contains the following testers for ports P1, P2, and P3:

Tester(P1,R1)

(T1) send A to P1

(T1) receive $\mathrm{X}$ from $\mathrm{P} 1$

(T4) send A to P1
Tester(P2,R1)

(T4) receive $Y$ from $P 2$
Tester(P3,R1)

(T3) send $\mathrm{C}$ to $\mathrm{P} 3$

(T3) receive $Z$ from $P 3$

(To improve readability, each send or receive statement in a tester is preceded with the name of the transition from which the statement is derived.) $\mathrm{R} 1$ is not pair-synchronizable since the input port of T3, which is P3, is not involved in T1 (i.e., P3 is neither the input port nor an output port of T1), and the input port of T4, which is P2, is not involved in T3. When an execution of M1 and the above testers starts, state $\mathrm{S} 1$ is the current state. When a transition of $\mathrm{S} 1$ is to be chosen, it is possible that messages $\mathrm{A}$ and $\mathrm{C}$ are available at ports $\mathrm{P} 1$ and $\mathrm{P} 3$, respectively. However, only $\mathrm{P} 1$ :A is valid for $\mathrm{S} 1$ and thus only transition $\mathrm{T} 1$ is eligible for $\mathrm{S} 1$. After the execution of T1, state $\mathrm{S} 2$ becomes the current state of M1. When a transition of S2 is to be chosen, it is possible that messages $\mathrm{A}$ and $\mathrm{C}$ are available at ports $\mathrm{P} 1$ and $\mathrm{P} 3$, respectively. However, only $\mathrm{P} 3: \mathrm{C}$ is valid for $\mathrm{S} 2$ and thus only transition T3 is eligible for S2. Although R1 is not pair-synchronizable, it does not have the problem of synchronizing inputs form different testers. Below we formally define the synchronization problem based on the use of port-based testers. In the next section, we show how to solve this synchronization problem.

Definition. Let $\mathrm{E}$ be a test sequence of an FSM M and let Tester(E) be the set of testers generated by algorithm Port_Tester_Gen according to E. During an execution of $\mathrm{M}$ and Tester(E), the port-based synchronization problem occurs when a state of $\mathrm{M}$ has two or more eligible transitions. (As a result of this problem, the execution of $M$ and Tester(E) is nondeterministic and may result in an abnormal termination.)

\section{PORT-SYNCHRONIZABLE TEST SEQUENCES OF AN FSM}

Definition. For a transition $\mathrm{T}$ in a transition sequence $\mathrm{E}$ of an FSM, its port-predecessor transition, denoted as PPT(T,E), is the closest predecessor of $T$ in $E$ that involves the input port of $T$. If such a predecessor of $T$ in $E$ does not exist, then PPT(T,E) is defined as null.

Let $T$ be a transition in a test sequence $E$ of an FSM M and let P:A be the input symbol of T. According to algorithm Port_Tester_Gen, the statement "send A to port P" appears in Tester(P,E). Consider the following two cases:

- PPT(T,E) is null. In this case, "send A to port $\mathrm{P}$ " is the first statement in Tester $(\mathrm{P}, \mathrm{E})$. During an execution of $\mathrm{M}$ and Tester(E), the statement "send $A$ to port $P$ " causes the port-based synchronization problem to occur if and only if $\mathrm{P}: \mathrm{A}$ is a valid input for one of the states entered before the head state of $\mathrm{T}$. 
- $\mathrm{PPT}(\mathrm{T}, \mathrm{E})$ is not null. In this case, "send A to port $\mathrm{P}$ " in Tester(P,E) appears immediately after the statements generated for PPT(T,E). Thus, T's input symbol arrives at M only after the completion of PPT(T,E). During an execution of M and Tester(E), the statement "send A to port $\mathrm{P}^{\prime \prime}$ causes the port-based synchronization problem to occur if and only if $\mathrm{P}: \mathrm{A}$ is a valid input for one of the states entered after the head state of PPT(T,E) and before the head state of $\mathrm{T}$.

Definition. For a transition $\mathrm{T}$ in a transition sequence $\mathrm{E}$ of an FSM, its port-predecessor interval, denoted as PPI(T,E), is defined as

the sequence of transitions in $\mathrm{E}$ after PPT(T,E) and before T, if PPT(T,E) $\neq$ null, or

the sequence of transitions in $\mathrm{E}$ before $\mathrm{T}$, if $\operatorname{PPT}(T, E)=$ null. Notes:

- If $\mathrm{T}$ is the first transition in E, then PPT(T,E) $=$ null and $\mathrm{PPI}(\mathrm{T}, \mathrm{E})=\boldsymbol{\varepsilon}$, where $\boldsymbol{\varepsilon}$ denotes the empty sequence.

- If PPT(T,E) is the immediate predecessor of $T$, then $\operatorname{PPI}(T, E)=\varepsilon$.

Definition. Let $\mathrm{T}$ be a transition in a transition sequence $\mathrm{E}$ of an FSM M. T is said to be portsynchronizable for (E,M), if

(a) $\operatorname{PPI}(T, E)$ is $\varepsilon$, or

(b) PPI(T,E) is not $\varepsilon$ and the input symbol of $T$ is invalid for the head state of any transition in PPI(T,E).

Note: Assume that T is port-synchronizable for (E,M). The arrival of T's input symbol before head( $\mathrm{T}$ ) becomes the current state of $\mathrm{M}$ does not create an additional eligible transition for the head state of any transition in PPI(T,E).

Definition. Let $\mathrm{E}$ be a transition sequence of an FSM M. E is said to be port-synchronizable for $\mathrm{M}$ if every transition in $\mathrm{E}$ is port-synchronizable for (E,M).

Consider the test sequence R2 = T1.T3.T4.T2.T3.T5 of M1. R2 is a postman tour of M1. Below we show the PPT and PPI of each transition in R2. Since T3 has two occurrences in R2, T3_1 and T3_2 denote the first and second occurrences of T3, respectively.

$\begin{array}{lllllll}\text { Transition T } & \text { T1 } & \text { T3_1 } & \text { T4 } & \text { T2 } & \text { T3_2 } & \text { T5 } \\ \text { PPT(T,R2) } & \text { null } & \text { null } & \text { T1 } & \text { T4 } & \text { T3_1 } & \text { T2 } \\ \text { PPI(T,R2) } & \boldsymbol{\varepsilon} & \text { T1 } & \text { T3_1 } & \boldsymbol{\varepsilon} & \text { T4.T2 } & \text { T3_1 }\end{array}$

$\mathrm{R} 2$ is not pair-synchronizable, but it is port-synchronizable for M1. (Note that no pairsynchronizable postman tours of M1 exist.) By applying algorithm Port_Tester_Gen to R2, we obtain the following testers for ports $\mathrm{P} 1, \mathrm{P} 2$, and $\mathrm{P} 3$ :

Tester(P1,R2)

(T1) send A to P1

(T1) receive $X$ from $P 1$

(T4) send A to P1

(T5) receive $\mathrm{X}$ from $\mathrm{P} 1$
Tester(P2,R2)

(T4) receive $Y$ from $\mathrm{P} 2$

(T2) send B to P2

(T2) receive $Y$ from $P 2$

(T5) send B to P2
Tester(P3,R2)

(T3_1) send $\mathrm{C}$ to $\mathrm{P} 3$

(T3_1) receive $\mathrm{Z}$ from $\mathrm{P} 3$

(T3_2) send $\mathrm{C}$ to $\mathrm{P} 3$

(T3_2) receive $\mathrm{Z}$ from $\mathrm{P} 3$ 
Consider another test sequence R3 = T1.T3.T5.T2.T3.T4 of M1. R3 is also a postman tour of M1. Below we show the PPT and PPI of each transition in R3.

$\begin{array}{lllllll}\text { Transition T } & \text { T1 } & \text { T3_1 } & \text { T5 } & \text { T2 } & \text { T3_2 } & \text { T4 } \\ \text { PPT(T,R3) } & \text { null } & \text { null } & \text { null } & \text { T5 } & \text { T3_1 } & \text { T5 } \\ \text { PPI(T,R3) } & \boldsymbol{\varepsilon} & \text { T1 } & \text { T1.T3_1 } & \boldsymbol{\varepsilon} & \text { T5.T2 } & \text { T2.T3_2 }\end{array}$

$\mathrm{R} 3$ is not port-synchronizable for M1 since

- $\operatorname{PPI}(\mathrm{T} 5, \mathrm{R} 3)=\mathrm{T} 1 . \mathrm{T} 3 \_1$ and the input symbol of T5 is valid for head(T1).

- $\quad \operatorname{PPI}(\mathrm{T} 4, \mathrm{R} 3)=\mathrm{T} 2 . \mathrm{T} 3 \_2$ and the input symbol of T4 is valid for head(T2).

Below we show some properties of pair- and port-synchronizable tests sequences of an FSM. Theorem 6.1. Let $\mathrm{E}$ be a test sequence for an FSM $M$.

(a) $\mathrm{E}$ is pair-synchronizable if and only if for each transition $\mathrm{T}$ in $\mathrm{E}, \mathrm{PPI}(\mathrm{T}, \mathrm{E})$ is $\varepsilon$.

(b) If $\mathrm{E}$ is pair-synchronizable, then $\mathrm{E}$ is port-synchronizable for $\mathrm{M}$. But the converse is not necessarily true.

(c) If $\mathrm{M}$ is completely specified, then $\mathrm{E}$ is pair-synchronizable if and only if $\mathrm{E}$ is portsynchronizable for $\mathrm{M}$.

Proof. This theorem follows the definitions of pair- and port-synchronizable test sequences of an FSM.

Q.E.D.

Theorem 6.2. Let $\mathrm{E}$ be a port-synchronizable test sequence of an FSM M and let Tester(E) be the set of testers generated by algorithm Port_Tester_Gen according to E.

(a) Any execution of $\mathrm{M}$ and Tester(E) is deterministic and successful. Thus, the port-based synchronization problem never occurs during any execution of $M$ and Tester(E).

(b) At any time during an execution of $\mathrm{M}$ and Tester(E), two or more ports of $\mathrm{M}$ may contain input messages.

(c) If $E$ is pair-synchronizable, then at any time during an execution of $\mathrm{M}$ and $\mathrm{Tester}(\mathrm{E})$, at most one port of $\mathrm{M}$ contains input messages.

(d) $\mathrm{M}$ does not contain a port-synchronizable test sequence $\mathrm{F}$ such that $\mathrm{F} \neq \mathrm{E}$ and Tester(F)=Tester(E).

(The proof is omitted.)

One interesting question is whether there exists a test sequence $E$ of an FSM M such that $E$ is not port-synchronizable for $\mathrm{M}$, but any execution of $\mathrm{M}$ and Tester(E) is deterministic. The following theorem says such a test sequence of $M$ does not exist. Based on this theorem, the definition of a port-synchronizable test sequence of $M$ provides a necessary and sufficient condition under which a test sequence of $\mathrm{M}$ does not have the synchronization problem. To prove this theorem, we consider the delayed execution of $\mathrm{M}$ and Tester(E), which means that during an execution of $M$ and Tester(E), the selection of a transition of the current state of $M$ is delayed until (a) each tester in Tester(E) either has completed or is blocking on a receive, and (b) all messages sent by testers in Tester(E) have arrived at ports of M. Obviously, the delayed execution of $M$ and Tester(E) is deterministic if and only if any execution of $M$ and Tester(E) is deterministic. 
Theorem 6.3. Let $\mathrm{E}$ be a test sequence of an FSM M and let Tester(E) be the set of testers generated by algorithm Port_Tester_Gen according to $\mathrm{E}$. $\mathrm{E}$ is port-synchronizable for $\mathrm{M}$ if and only if any execution of $\mathrm{M}$ and Tester(E) is deterministic.

(The proof is omitted.)

\section{GENERATION OF PORT-SYNCHRONIZABLE TEST SEQUENCES OF AN FSM}

In this section, we briefly discuss possible approaches to extending a test sequence generation method for an FSM in order to generate port-synchronizable test sequences. One approach is to apply the concept of backtracking. Assume that during the application of a test sequence generation method to an FSM M, E is the partial test sequence generated so far and transition T is allowed to follow E. We need to determine whether T is port-synchronizable for E.T with respect to $M$. If the answer is yes, then we append $T$ to the end of $E$ and continue the original test sequence generation procedure. If the answer is no, then we try to find another transition, say $\mathrm{T}$, that is allowed to follow $\mathrm{E}$, and then we determine whether $\mathrm{T}^{\prime}$ is port-synchronizable for E. $\mathrm{T}^{\prime}$ with respect to $M$. We repeat this process until such a transition is found. If no such transition exists for $\mathrm{E}$, then we replace the last transition in $\mathrm{E}$ with another transition and repeat the same process. Eventually either we find one port-synchronizable test sequence of $\mathrm{M}$, or we conclude that no port-synchronizable test sequence of $\mathrm{M}$ exists, according to the original test sequence generation method. The concept of backtracking was also used in the generation of pairsynchronizable test sequences (Sarikaya and Bochmann, 1984) (Luo et al., 1993).

Some test sequence generation methods involve the generation of two types of transition sequences for a state $\mathrm{S}$ of an FSM M:

- transfer sequences for $S$, which start from the initial state of $M$ and reach at $S$.

- state identification or validation sequences for $\mathrm{S}$, which start from $\mathrm{S}$.

Assume that for a transition $\mathrm{T}$ of $\mathrm{M}$, a test sequence generation method has been extended to generate

- a set FS of port-synchronizable transfer sequences for head(T), and

- a set DS of port-synchronizable identification or validation sequences for tail(T).

We need to find one element of FS, say F, and one element of DS, say D, such that F.T.D is portsynchronizable for $\mathrm{M}$. To search for such a port-synchronizable test sequence, we have the following observations:

(a) For each element D of DS, let Null(D) be the set of transitions in D with their PPT being null. To determine whether F.T.D is synchronizable for $M$, we only need to determine whether T and transitions in Null(D) are synchronizable for (F.T.D, M). Transitions in D that are not in Null(D) can be ignored, since they can never create a port-base synchronization problem for F.T.D.

(b) Let $U$ be the shortest suffix of $F$ that involves the input ports of $T$ and transitions in Null(D) at least once. To determine whether F.T.D is port-synchronizable for $\mathrm{M}$, we only need to determine whether $\mathrm{T}$ and transitions in Null(D) are port-synchronizable for (U.T.D, M). The 
reason is that $\mathrm{T}$ and transitions in Null(D) have their PPT in $\mathrm{U}$.

Based on (b), we have developed algorithms for generating UIO-based port-synchronizable test sequences of an FSM.

\section{RESULTS OF EMPIRICAL STUDIES}

In this section, we describe the results of our empirical studies on pair- and port-synchronizable test sequences. We used a number of FSM-based protocols in our empirical studies. For each FSM-based protocol $\mathrm{M}$, we applied the following steps:

(a) used tsg, a test sequence generation tool, to generate a set $\mathrm{S}$ of test sequences of $\mathrm{M}$.

(b) for each test sequence $E$ in $S$, determined whether $E$ is pair-synchronizable, not pairsynchronizable, but port-synchronizable for $\mathrm{M}$, or not port-synchronizable for $\mathrm{M}$.

The tsg (test sequence generation) tool was obtained from the Department of Computer Science at University of British Columbia. For a given FSM, tsg can generate test sequences according to the D-method (Kohavi, 1978), UIO-method (Sabnani and Dahbura, 1988), and W-method (Chow, 1978). For the input FSM, the tool inserts a reset transition for each state. If the input FSM is not completely specified, the tool inserts a self-loop transition for each unspecified input of a state. Each of the inserted transitions has no output.

We applied tsg to generate UIO-based test sequences for a number of FSM-based protocols. For each transition $\mathrm{T}$ in a given FSM, tsg generated one or more UIO-based test sequences, each starting with a reset transition, followed by u.T.v, where $u$ is a transition sequence from the initial state to the head state of $\mathrm{T}$ and $\mathrm{v}$ is a UIO sequence for the tail state of $\mathrm{T}$. After a set $\mathrm{S}$ of UIO-based test sequences for an FSM was generated by tsg, $\mathrm{S}$ was modified as follows:

(a) The test sequences in $\mathrm{S}$ for inserted transitions (i.e., reset transitions and the self-loop transitions for unspecified inputs) were deleted.

(b) For each of the remaining test sequences in $\mathrm{S}$, the inserted transitions in this test sequence, if they existed, were deleted, and if the resulting test sequence contained zero or one transition, then this test sequence was deleted.

(c) After step (b), if two or more test sequences in S were identical, then only one of them was kept in $\mathrm{S}$.

The following algorithm was used to determine whether a test sequence is pair-synchronizable, and if not, whether it is port-synchronizable for a given FSM M.

let $\mathrm{E}=\mathrm{E} 1 . \mathrm{E} 2$.....En be a test sequence of $\mathrm{M}$;

pair_flag := true; $\quad / *$ to indicate whether E is pair-synchronizable */

port_flag := true; $\quad / *$ to indicate whether $E$ is port-synchronizable for $M * /$

for $\mathrm{i}=2,3, \ldots, \mathrm{n}$ until port_flag $=$ false do begin

visit transition $\mathrm{Ei}$;

assume that the input symbol of $\mathrm{Ei}$ is $\mathrm{P}: \mathrm{A}$; 
if port $\mathrm{P}$ is not involved in $\mathrm{E}(\mathrm{i}-1)$ then begin

pair_flag := false;

ppt_found := false; $\quad / *$ to indicate whether PPT(Ei,E) has been found */

for $\bar{j}=\mathrm{i}-1, \mathrm{i}-2, \ldots, 1$ until port_flag $=$ false or ppt_found $=$ true do begin

visit transition $\mathrm{Ej}$;

if port $\mathrm{P}$ is involved in $\mathrm{Ej}$

then ppt_found := true;

end; end; end;

else if $\mathrm{P}: \mathrm{A}$ is a valid input for head(Ej) then port_flag := false;

After the completion of the above algorithm, if pair_flag is true, then $\mathrm{E}$ is pair-synchronizable. Otherwise, if port_flag is true, then $\mathrm{E}$ is not pair-synchronizable, but port-synchronizable for $\mathrm{M}$. If port_flag is false, then $\mathrm{E}$ is not port-synchronizable for $\mathrm{M}$.

The following table shows some of the results of our empirical studies.

\begin{tabular}{|c|c|c|c|c|}
\hline protocol name & test_seq_\# & pair_\# & port_\# & non_port_\# \\
\hline T-class-0 & 55 & $45(82 \%)$ & $52(95 \%)$ & $3(5 \%)$ \\
\hline T-class-4 & 111 & $85(76 \%)$ & $89(80 \%)$ & $22(20 \%)$ \\
\hline Q931 & 50 & $20(40 \%)$ & $48(96 \%)$ & $2(4 \%)$ \\
\hline
\end{tabular}

where

- test_seq_\# is the total number of UIO-based test sequences generated by tsg for the corresponding protocol.

- pair_\# is the number of pair-synchronizable test sequences.

- port_\# is the number of port-synchronizable test sequences.

- non_port_\# is the number of non-port-synchronizable test sequences.

- T-class-0 refers to the transport class 0 protocol (Sarikaya and Bochmann, 1984) which has 4 states and 21 transitions.

- T-class-4 refers to the transport class 4 protocol (Sidhu and Leung, 1989), which has 15 states and 60 transitions.

- Q931 refers to the ISDN Q931 protocol (Zhu and Chanson, 1994), which has 8 states and 31 transitions.

Both T-class-4 and Q931 contain multiple types of timeout signals, which were treated as inputs from a unique port in our empirical studies.

The above table show that for the set of UIO-based test sequences generated by tsg for a protocol, the percentage of pair-synchronizable test sequences ranges from $40 \%$ to $82 \%$ and that the percentage of port-synchronizable test sequences ranges from $80 \%$ to $96 \%$. The increase of percentage of synchronizable test sequences due to the definition of a port-synchronizable test sequence ranges from $4 \%$ to $56 \%$. These results indicate that the use of port-synchronizable test sequences may significantly increase the number of synchronizable test sequences of an FSM. 


\section{CONCLUSIONS}

In this paper, we have defined port-synchronizable test sequences of an FSM with multiple ports and shown that this definition is a necessary and sufficient condition under which a test sequence of an FSM does not have the synchronization problem. Our empirical studies show that an FSM may contain many port-synchronizable test sequences that are not pair-synchronizable. The use of port-synchronizable test sequences in protocol testing makes more test sequences to become synchronizable.

As mentioned in Section 4, group-based testing of an FSM M is to divide the ports of M into groups and allows the testers for ports in the same group to communicate with each other in order to synchronize the arrivals of inputs at $\mathrm{M}$. For a given definition of groups of ports of $\mathrm{M}$, we have defined a necessary and sufficient condition under which a test sequence of $\mathrm{M}$ either does not have the synchronization problem or can have the synchronization problem solved by allowing communication between testers for ports of $M$ in the same group (Tai and Young 1995). For a test sequence of $M$ that satisfies this condition, we have shown how to construct a set of testers with minimum communication between them. Also, we have studied the impact of timeout transitions on port- and group-synchronizable test sequences.

The use of the multi-port FSM model to specify communication protocols is increasing. We are currently investigating the generation of port- and group-synchronizable test sequences and the fault detection capability of port- and group-based testing.

\section{ACKNOWLEDGMENTS}

The authors wish to thank Dan Duvarney for his effort in carrying out empirical studies. Also, the authors are grateful to Pramod Koppol for his comments and to S. T. Chanson and J. Zhu for providing the tsg tool.

\section{REFERENCES}

Boyd, S. and Ural, H. (1991) The synchronization problem in protocol testing and its complexity, Information Processing Letters, Vol. 40, 131-6.

Carver, R. H. and Tai, K. C. (1991) Replay and testing for concurrent Programs, IEEE Software, Vol. 8, No. 2, 66-74.

Chen, W. H., Lu, C. S., Chen, L. and Tang, J. T. (1990) Synchronizable protocol test generation via the duplex technique, Proc. IEEE INFOCOM, 561-3.

Chen, W. H. and Ural, H. (1995) Synchronizable test sequences based on multiple UIO sequences, IEEE/ACM Trans. Network, Vol.3, No. 2, 152-7.

Chow, T. S. (1978) Testing software modeled by finite-state machines, IEEE Trans. Software Eng., Vol. SE-4, No. 3, 178-87. 
Guyot, S. and Ural, H. (1995) Synchronizable checking sequences based on UIO sequences, Proc. Protocol Test Systems VIII.

Kohavi, Z. (1978) Switching and Finite Automata Theory. 2nd edition, McGraw-Hill.

Luo, G., Dssouli, R., Bochmann, G. v., Venkataram, P. and Ghedamsi, A. (1993) Generating synchronizable test sequences based on finite state machine with distributed ports, Proc. Protocol Test Systems VI, 139-153.

Sarikaya, B. (1993) Principles of Protocol Engineering and Conformance Testing, Ellis Horwood Limited.

Sarikaya, B. and Bochmann, G. v. (1984) Synchronization and specification issues in protocol testing, IEEE Trans. on Communications, Vol. 32, No.4, 389-95.

Sabnani, K. and Dahbura, A. (1988) A protocol test generation procedure, Computer Networks and ISDN Systems, Vol. 15, no. 4, 285-97.

Sidhu, D. P. and Leung, T. K. (1989) Formal Methods for protocol testing: A detailed study, IEEE Trans. on Software Engineering., Vol. 15, no. 4, 413-26.

Tai, K. C. and Ahuja, S. (1987) Reproducible testing of communication software, Proc. IEEE Inter. Conf. on Computer Software and Applications (COMPSAC), 331-7.

Tai, K. C., Carver, R. H., and Obaid, E. E. (1991) Debugging concurrent Ada programs by deterministic execution, IEEE Trans. Soft. Eng., Vol. 17, No. 1, 45-63.

Tai, K. C., and Carver, R. H. (1995) Testing of Distributed Programs, in Handbook of Parallel and Distributed Computing (ed. A. Zoyama), McGraw-Hill.

Tai, K. C., and Young, Y. C. (1995) Synchronizable test sequences of finite state machines, Technical Report, TR-95-11, Dept. of Computer Science, North Carolina State University.

Tarnay, K. (1991) Protocol Specification and Testing, Plenum Press.

Turner, K. J. (1993) Using Formal Description Techniques: An Introduction to Estelle, Lotos, and $S D L$, Wiley.

Ural, H. and Wang, Z. (1993) Synchronizable test sequence generation using UIO sequences, Computer Communications, Vol. 16, No. 10, 653-61.

Zhu, J. and Chanson, S. T. (1994) Toward evaluating fault coverage of protocol test sequences, Proc. Protocol Specification, Testing, and Verification XIV, 130-44.

\section{BIOGRAPHY}

Kuo-Chung Tai is a Professor in the Computer Science Department at North Carolina State University. He has published papers in the areas of software engineering, distributed systems, programming languages, and compiler construction. His current research interests include analysis, testing, and debugging of sequential and concurrent software. He received his Ph.D. degree in Computer Science from Cornell University in 1977. From 1989 to 1991, he served as the director of Software Engineering Program at the National Science Foundation. He is an associate editor of Journal of Computer Languages, Inter. Journal of Software Engineering and Knowledge Engineering, and Inter. Journal of Computer and Software Engineering.

Yu-Chiou Young is a Ph.D. student in the Computer Science Department at North Carolina State University. He is also a full-time employee of IBM in Raleigh, North Carolina. 